\title{
Normatividad sobre Arquitectura Bibliotecaria en México, elemento imprescindible para la oferta de servicios de información
}

\author{
Celia Mireles Cárdenas * \\ J. Alberto Arellano Rodríguez **
}

Artículo recibido:

21 de octubre de 2013.

Artículo aceptado:

30 de abril de 2014.

\section{Resumen}

El edificio de una biblioteca constituye la primera imagen que el público se forma con relación a los servicios que puede esperar de ella y llega a representar un símbolo del nivel cultural, económico y social de una institución, ciudad o país, por lo que estudiar los elementos que influyen en su edificación, y por ende en la prestación de servicios de información, se constituye en una labor que no puede ser postergada en las sociedades actuales marcadas, entre otros factores, por el impacto del desarrollo tecnológico, el cambio climático, la globalización cultural, la seguridad social, así como el cambio de valores y costumbres que guían el comportamiento del ser humano.

* Universidad Autónoma de San Luis Potosí, México. cmireles@uaslp.mx

** Universidad Autónoma de Yucatán, México. alberto.arellano@uady.mx 
En México es poca la literatura que refiere al número y características con que cuentan estos edificios, por lo que el presente documento describe la normativa mexicana que trata sobre la construcción de bibliotecas a partir de la identificación de la legislación, políticas, actores e instituciones que han abordado el tema, de tal forma que al final se presenta la problemática detectada como un primer acercamiento para la elaboración de propuestas viables, en beneficio de una mejor oferta de servicios de información.

Palabras clave: Arquitectura de bibliotecas; Edificios para bibliotecas; Normas para la construcción de bibliotecas; Servicios de información y espacios.

\section{Abstract}

The need for library architecture standards in Mexico to ensure the provision of information services

Celia Mireles-Cárdenas and J. Alberto Arellano-Rodri-

guez

The building housing a library is the first image the public grasps concerning what he can expect to receive inside. Moreover, the library building is a symbol of the cultural, economic, and social development of an institution, city or country. Gaining a better awareness of the influences involved in library construction and, consequently, the information services it provides should not be put off by societies impacted by technological development, climate change, cultural globalization, social security, as well as shifting values and customs informing the behavior of human beings. Mexico has scant literature addressing the features library buildings. This study, therefore, analyzes Mexican library construction regulations, identifying current law, policies, and agencies and institutions holding stakes in this field. Finally, these issues are discussed terms of how information services might feasibly be improved.

Keywords: Library Architecture; Library Buildings; Regulations for Library Construction; Information Services; Information Spaces. 


\section{INTRODUCCIÓN}

$\Lambda$ partir de la oferta de servicios de información pertinentes, las bibliote- cas pueden ser instrumentos de cambio en la sociedad y contribuir al desarrollo económico, científico y social. Para lograrlo, un elemento fundamental es contar con espacios suficientes, adecuados y flexibles para el estudio, la investigación, la cultura y el ocio, que permitan una eficiente disponibilidad de los recursos de información, tanto impresos como digitales.

En 1974, Fernández de Zamora escribió para la Encyclopedia of library and Information Science que la arquitectura de bibliotecas en México era casi inexistente. ${ }^{1}$ En la actualidad la situación ha cambiado y se tiene un panorama, si bien no ideal, en el que se observa un incesante crecimiento en la construcción de bibliotecas en el país, de todo tipo y tamaño, principalmente en los sectores público y universitario.

Precisamente, la experiencia lograda por instituciones universitarias en las últimas cuatro décadas las convierte en el referente natural para la construcción de otro tipo de bibliotecas, como son las públicas estatales y municipales; casos recientes son los de la Universidad Autónoma de Yucatán (UADY) con la construcción de la Biblioteca Estatal de Ciencias ${ }^{2}$ y la Universidad de Guadalajara (UdeG) con la Biblioteca Pública del Estado de Jalisco, así como los proyectos de construcción presentados por los Rectores de la Universidad Autónoma de San Luis Potosí (UASLP) ${ }^{3}$ y de la Universidad Autónoma de Ciudad Juárez ${ }^{4}$ para construir bibliotecas públicas en los Centros Históricos de las respectivas ciudades.

Sin embargo, a pesar del avance obtenido en la construcción de espacios destinados para la oferta de servicios bibliotecarios en México, poco se conoce de la evolución en los procesos de construcción, de las características que tienen los espacios y de su impacto en los usuarios, así como en los trabajadores de las bibliotecas. Con relación a los principios que se deben tomar

1 Rosa María Fernández de Zamora, “Construcción de Nuevas Bibliotecas en México, 1981”, pp. 328-330.

2 Roberto Ancona Riestra, "Biblioteca Estatal de Ciencias. Parque Científico y Tecnológico de Yucatán”.

3 Manuel Fermín Villar Rubio, "Impacto social de los servicios bibliotecarios en la Universidad Autónoma de San Luis Potosí”.

4 Propuesta de trabajo, punto 8. Divulgación y difusión de los saberes, establece la intención de que "[...] a partir del trabajo cooperativo de investigadores, profesores y empresarios culturales que laboran dentro de la UACJ, se promoverá la realización de un proyecto para mejorar en el mediano plazo la infraestructura cultural de la ciudad. Aunque se realizarán los estudios de factibilidad pertinentes, un primer proyecto será la construcción de una gran Biblioteca Pública en el Centro Histórico”. Ricardo Duarte Jáquez, La Universidad que queremos... Una propuesta de trabajo para consolidar la calidad académica y la mejora de la gestión en el periodo rectoral 2012-2018. Resumen, p. 18. 
en cuenta para la construcción de estos edificios, se detecta que la normativa utilizada presenta características relacionadas con traducción literal, desactualización, visión parcial en la interpretación y adaptación por parte de bibliotecarios, responsables de las bibliotecas u organismos de acreditación y certificación ajenos a la profesión; aspectos entre otros que conllevan a una problemática relacionada con la funcionalidad y pertinencia de la normativa utilizada para la planeación, diseño, construcción, remodelación y uso de espacios bibliotecarios en el país.

Recordemos que la normativa se refiere al "conjunto de normas aplicables a una determinada materia o actividad", 5 mientras que una norma

[...] es un patrón, modelo o regla que debe seguirse o ajustarse a alguna actividad. Son especificaciones técnicas que contienen un conjunto de condiciones a ser cumplidas ya que se han establecido por alguna autoridad o personal autorizado. En las normas se establecen cantidades, modelos, métodos o unidades de medida que deben de ser adoptadas como el mínimo común o como ejemplo a seguir. También puede considerarse como un tipo de plan, que sirve como unidad de medida establecida, como criterio, parámetro o nivel de referencia. ${ }^{6}$

Es por ello que, con base en la experiencia acumulada en la construcción de bibliotecas, desde el 2010 se ha establecido un grupo de trabajo conformado por Manuel Fermín Villar Rubio, Rector de la UASLP; Roberto Ancona Riestra, Jefe de Proyectos de la Facultad de Arquitectura de la UADY; J. Alberto Martínez Arellano, Coordinador General del Sistema de Bibliotecas de la UADY; José Alfredo Verdugo Sánchez, Director de Bibliotecas de la Universidad Autónoma de Baja California Sur (UABCS); Juan Ángel Vázquez Martínez, Director de Bibliotecas de la Universidad Autónoma del Carmen (UNACAR) y Celia Mireles Cárdenas, profesora e investigadora de la UASLP, con el objetivo de conformar un marco general a partir del cual se conjunte, revise y actualice la normativa existente para la planeación, el diseño y la construcción de bibliotecas universitarias en México, en búsqueda de una mejor oferta de servicios de información y la optimización de los recursos destinados para ello.

De esta forma, a partir de la revisión y análisis bibliográfico así como de los resultados de las reuniones de trabajo realizadas en la UASLP y en la UADY, este documento tiene como objetivos:

5 Diccionario de la Lengua Española, s. v. "normativa”. Consultado en: http://lema.rae.es/drae /?val=normativa

6 María Esther Mendoza Zavala, Memoria de la elaboración de normas para espacios físicos de las Bibliotecas del Sistema Bibliotecario de la UNAM, p. 9. 
1. Describir la normativa mexicana que refiere a la construcción de bibliotecas, a partir de la identificación de la legislación, políticas, actores e instituciones que han abordado el tema.

2. Identificar la problemática existente como un primer acercamiento para la elaboración de propuestas viables que incidan en una mejor oferta de servicios de información.

\section{LEGISLACIÓN MEXICANA E INFRAESTRUCTURA BIBLIOTECARIA}

Los lineamientos a partir de los cuales se planean, diseñan, construyen, remodelan y en su caso restauran los espacios bibliotecarios públicos y educativos tienen su fundamento en el artículo 3o. de la Constitución Política de los Estados Unidos Mexicanos y se relacionan directa o indirectamente con otros artículos del mismo ordenamiento, como el 73, fracción XXV, que señala el establecimiento y sostenimiento de bibliotecas en toda la República como facultad del Congreso, y el 123, fracción XV, del "Título Sexto. Del Trabajo y de la Previsión Social", que dispone la obligación de contemplar los preceptos de higiene y seguridad en las instalaciones laborales y la adopción de medidas adecuadas para prevenir accidentes y enfermedades en el uso de máquinas, instrumentos y materiales de trabajo. ${ }^{7}$

Recordemos en este punto que las bibliotecas como centros públicos de trabajo se encuentran sujetas a la legislación laboral del país, máxime cuando es conocido que algunas actividades bibliotecarias implican riesgos que pueden dañar la salud y provocar heridas y lesiones por el contacto que se tiene con polvo, hongos, insecticidas y con el traslado y manejo del material bibliográfico, entre otros, por lo que el edificio debe contemplar aspectos de seguridad en escaleras, pisos, muros, techos, plafones, dispositivos de seguridad, salidas de emergencia y equipos contra incendios, ya que iluminación, ventilación, ruido y temperatura inadecuados pueden afectar la salud de los trabajadores de la biblioteca. ${ }^{8}$

Por su parte, la Ley General de Educación (1993) da pauta a la realización de diferentes acciones que han regulado el establecimiento y construcción de bibliotecas a lo largo de varias décadas en el país, lo que ha fortalecido el sistema educativo nacional que comprende la educación inicial, la educación superior, la educación especial y la educación para adultos. De esta forma, la Ley

7 De aquí en adelante, las leyes federales que se mencionen se consultaron en http://www.diputados.gob.mx/LeyesBiblio/index.htm

8 Rosa María Martínez Rider, Higiene y seguridad en bibliotecas públicas de la ciudad de San Luis Potosí. 
establece en su artículo 14, fracción VI, que entre las atribuciones referidas a las autoridades educativas federal y locales, está la de "Prestar servicios bibliotecarios a través de bibliotecas públicas, a fin de apoyar al sistema educativo nacional, a la innovación educativa y a la investigación científica, tecnológica y humanística”.

A su vez, la Ley General de la Infraestructura Física Educativa (2008) ${ }^{9}$ establece en su artículo 2o. que su objetivo es el de "[...] regular la infraestructura física educativa al servicio del sistema educativo nacional, estableciendo los lineamientos generales para: I. La construcción, equipamiento, mantenimiento, rehabilitación, reforzamiento, reconstrucción y habilitación de inmuebles e instalaciones destinados al servicio del sistema educativo nacional”, mientras que en su capítulo IV brinda las bases para la creación del Instituto Nacional de la Infraestructura Física Educativa (INIFED), organismo descentralizado de la Secretaría de Educación Pública, cuya finalidad es la de:

[...] fungir como un organismo con capacidad normativa, de consultoría y certificación de la calidad de la infraestructura física educativa del país y de construcción [...], y desempeñarse como una instancia asesora en materia de prevención y atención de daños ocasionados por desastres naturales, tecnológicos o humanos en el sector educativo.

Para el cumplimiento de lo establecido en el párrafo anterior, el Instituto considerará en todo momento las características particulares de cada región del país, con base en su riqueza y diversidad. ${ }^{10}$

Para dar cumplimiento a lo anterior, el Instituto ha generado diversos documentos como normas, reglamentos, manuales, lineamientos y especificaciones técnicas para la realización de estudios, proyectos, construcción de instalaciones, funcionamiento, aseguramiento de la calidad, seguridad, salud y medio ambiente en los espacios educativos nacionales, como son el Reglamento de Ley que crea al Comité Administrador del Programa Federal de Construcción de Escuelas (CAPFCE) y el Reglamento de Seguridad, Salud y Medio Ambiente en el Trabajo.

Respecto a la conservación y restauración es común encontrar que espacios destinados a la oferta de servicios bibliotecarios se encuentran alojados en edificios con valor histórico o valioso por su significación en el contexto urbano, además de que por la relevancia del quehacer universitario, las construcciones de estas entidades públicas pasan a formar parte de la infraestruc-

9 Última reforma publicada DOF 14-03-2013.

10 Artículo 16 de la Ley General de la Infraestructura Física Educativa. El portal del Instituto Nacional de la Infraestructura Física Educativa es http://www.inifed.gob.mx/ 
tura educativa nacional. Otro aspecto importante es que con frecuencia las universidades son designadas como depositarias de legados históricos de cada región o ciudad, con lo que además de su misión educativa contribuyen a la conservación y difusión del patrimonio histórico del país. Tanto edificios como colecciones documentales se rigen bajo las siguientes dos leyes que especifican la ejecución de obras de construcción, reconstrucción, modificación, adaptación, conservación y mantenimiento de inmuebles federales.

Es así como la Ley Federal sobre Monumentos y Zonas Arqueológicos, Artísticos e Históricos (1972) señala en su artículo 2o. que "es de utilidad pública, la investigación, protección, conservación, restauración, y recuperación de los monumentos arqueológicos, artísticos e históricos y de las zonas de monumentos"; mientras que el artículo 36, fracción I, señala la naturaleza de los muebles e inmuebles considerados en esta Ley, entre ellos, los destinados a "[...] la educación y a la enseñanza, a fines asistenciales o beneficios; al servicio y ornato públicos y al uso de autoridades civiles y militares”. 11

Por su parte la Ley General de Bienes Nacionales (2004) señala en su artículo 4o. que están sujetos al régimen de dominio público de la Federación:

[...]

VI. Los inmuebles federales que estén destinados de hecho o mediante un ordenamiento jurídico a un servicio público y los inmuebles equiparados a éstos conforme a esta Ley; [...]

VIII. Los inmuebles federales considerados como monumentos arqueológicos, históricos o artísticos conforme a la ley de la materia o la declaratoria correspondiente; [...]

XIV. Las pinturas murales, las esculturas y cualquier obra artística incorporada o adherida permanentemente a los inmuebles sujetos al régimen de dominio público de la Federación; ${ }^{12}$

Diversas bibliotecas en el país se encuentran en esta situación, siendo referente obligado la Biblioteca Central de la UNAM.

En materia propiamente bibliotecaria, la Ley General de Bibliotecas (1988), en el Capítulo 1. Disposiciones generales, establece la observancia de los gobiernos federal, estatal y municipal para el establecimiento, sostenimiento y organización de las bibliotecas públicas, impulsando el equipamiento, mantenimiento y actualización permanente de un área de servicios de cómputo y los servicios culturales complementarios que a través de éstas se otorguen. En el Capítulo 2. De la Red Nacional de Bibliotecas Públicas, 
artículo 8, fracciones IV y IX, menciona la obligación de asegurar de modo integral y conservar el buen estado de las instalaciones, el equipo y acervo bibliográfico, por lo que es necesario dotar a sus bibliotecas de los locales y del equipo necesario para la prestación de los servicios bibliotecarios. ${ }^{13}$

En el caso de la Ley de Fomento para la lectura y el libro (2008), el Capítulo 1, artículo 4, fracción III menciona que la ley tiene por objeto: "Fomentar y apoyar el establecimiento y desarrollo de librerías, bibliotecas y otros espacios públicos y privados para la lectura y difusión del libro".

Con relación a la construcción y/o adecuación de los espacios físicos, estas dos leyes no establecen criterios ni referencias directas sobre cuáles deben ser las características de estos espacios.

\section{Políticas gubernamentales, FACTOR DE IMPUlso PARA LA CONSTRUCCIÓN DE BIBLIOTECAS}

México cuenta con una rica historia bibliotecaria desde la época prehispánica hasta nuestros días. Fernández de Zamora menciona que, bajo el modelo educativo implementado por José Vasconcelos, es con la inauguración de la biblioteca Miguel de Cervantes Saavedra el 28 de enero de 1924 cuando se puede hablar de un edificio construido expresamente para ello y considerarlo como una biblioteca moderna. ${ }^{14}$

En este rubro destacan por su importancia las experiencias obtenidas en el ámbito universitario, las cuales inician en 1950 con la construcción de la Biblioteca Central de la Universidad Nacional Autónoma de México (UNAM), que abre sus servicios al público el 5 de abril en $1956 .{ }^{15}$ Posteriormente, la Universidad Veracruzana (UV) se convierte en la segunda universidad que construye un edificio para tal fin cuando en 1960 inaugura la Biblioteca Central en el circuito Gonzalo Aguirre Beltrán. ${ }^{16}$ Más adelante, diversas universidades públicas y privadas edificaron importantes construcciones destinadas a la oferta de servicios bibliotecarios. ${ }^{17}$ En este contexto resalta la construcción del edificio de la Biblioteca Daniel Cosío Villegas de El Colegio de México (COLMEX), que fue inaugurado el 23 de septiembre de 1976, el

13 Última reforma aplicada DOF 23-06-2009.

14 Rosa María Fernández de Zamora, "Las bibliotecas y sus edificios en México: Una mirada histórica", p. 63.

15 Biblioteca Central. Libros, muros y murales. 50 aniversario, p. 17; "Acerca de la Biblioteca. Historia”, disponible en http://bc.unam.mx/historia.html

16 USBI, pionera en México. Disponible en http://www.uv.mx/universo/395/central/central.html

17 Rosa María Fernández de Zamora reseña a grandes rasgos la situación sobre la construcción de bibliotecas en el país hasta 1980 en "Construcción de Nuevas Bibliotecas en México, 1981". 
cual se convirtió en referente nacional tanto por su estructura física como por las contribuciones realizadas por Ario Garza Mercado, experto y pionero en el país en materia de planeación de edificios.

La construcción de edificios bibliotecarios tiene un aumento considerable a partir de la década de 1970 con el impulso de los diferentes planes y programas de gobierno en materia educativa, los cuales en mayor o menor medida destinan recursos para tal fin. En palabras de Fernández de Zamora, “[...] después de la época de Vasconcelos, es en el sexenio de 1976-1982 cuando las bibliotecas vuelven a florecer y cobrar importancia", ${ }^{18}$ impulsadas en gran medida por la Dirección General de Bibliotecas de la SEP.

De esta forma, en dicha década las políticas gubernamentales propiciaron una expansión de las universidades mexicanas mediante reformas estructurales para la transformación de las Instituciones de Educación Superior (IES), lo que originó un incremento de los servicios educativos del nivel medio superior y superior, pero a su vez, un deterioro académico y el abandono de los servicios bibliotecarios dado que el incremento de alumnos superó a la planta académica y a los servicios de apoyo, como eran considerados la biblioteca y los servicios de información. ${ }^{19}$

Vale la pena mencionar el Programa de Desarrollo Nacional de los Servicios Bibliotecarios y de Información (PRODENASBI), coordinado durante 1979-1980 por la Dirección General de Publicaciones y Bibliotecas de la SEP, que consistió en formular un programa de acción congruente y acorde a la realidad del país en aquella época y que propició la elaboración y publicación en 1984 de los Indicadores para Bibliotecas Públicas. Participaron en el estudio la Dirección de Edificios de la propia Secretaría, asesorados por el Centro Regional de Construcciones Escolares para América Latina y el Caribe (CONESCAL) y asociaciones bibliotecarias como la Asociación Mexicana de Bibliotecas A. C. (AMBAC), la Asociación de Bibliotecarios de Instituciones de Enseñanza Superior y de Investigación (ABIESI), y el Colegio Nacional de Bibliotecarios (CNB).

A partir del objetivo que consistió en "contribuir al desenvolvimiento socio-económico, científico, técnico, educativo y cultural del país, por medio del establecimiento de unidades de información suficientes en calidad y cantidad, adecuadas a las necesidades y características del mismo", en el PRODENASBI se desglosaron los siguientes objetivos específicos que tienen que ver con los espacios:

18 Ibid., p. 331.

19 Egbert J. Sánchez Vanderkast, Políticas de Información en las Universidades Públicas Estatales, p. 72 . 
- Racionalizar y distribuir armónicamente en el territorio nacional, las unidades de información con base en un sistema de ciudades, tal como lo anota el Plan Nacional de Desarrollo Urbano.

- Construir, sustituir, remodelar, ampliar y conservar las unidades de información, con base en el diagnóstico de la situación actual y de los requerimientos futuros.

- Mejorar la calidad y cantidad de mobiliario y equipo de unidades de información. ${ }^{20}$

En la década de 1980 se observa un estancamiento aun cuando las políticas generadas se orientaron hacia la búsqueda de la consolidación de la calidad educativa, entre otros factores por la diversificación en la oferta de áreas académicas y la evolución de la matrícula por áreas de conocimiento.

Para la década de 1990, el énfasis en la educación fue hacia la planeación, la distribución por áreas de conocimiento y los cambios de matrícula por género, entre otros aspectos que caracterizaron el quehacer universitario en esos años. De esta forma las políticas educativas se orientaron hacia el fomento de la calidad educativa a través de la modernización de la educación. En este periodo se establecieron los cimientos para tener mejores bibliotecas universitarias y servicios de información a nivel superior, los cuales quedaron plasmados en los programas de modernización educativa, de desarrollo nacional y en el Programa Nacional de Educación.

El programa sectorial que marcó el rumbo hacia el fortalecimiento en materia de infraestructura fue el Programa para la Modernización Educativa (PME) 1989-1994, lo anterior a pesar de que los diferentes rubros y documentos que lo integran no definen un concepto claro sobre la biblioteca, ya que la mencionan de diferentes formas, algunas de las cuales siguen vigentes en los distintos niveles educativos:

a) Cuando se refiere a las bibliotecas en educación superior universitaria, utiliza Sistema de Bibliotecas.

b) Cuando habla de la perspectiva de la educación tecnológica superior, utiliza centros de información y documentación, y bibliotecas.

c) Cuando se dirige a estos apoyos desde el posgrado e investigación científica y humanística los denomina acervos informativos, bibliotecas y los sistemas de información y documentación. ${ }^{21}$

20 PRODENASBI Programa de Desarrollo Nacional de los Servicios Bibliotecarios y de Información, p. 17. 21 Ibid., p. 71. 
En esta misma década, bajo el Programa de Desarrollo Educativo (PDE) 1994-2000 se enfatiza el fomento de la calidad académica y la modernización de la infraestructura de apoyo con el Fondo para Modernizar la Educación Superior (FOMES), actualmente conocido como Programa Integral de Fortalecimiento Institucional (PIFI), en donde se incluye a las bibliotecas.

Asimismo, con la finalidad de mejorar el desempeño académico e institucional y por consiguiente su calidad, se construyeron diferentes entidades de evaluación, algunas de las cuales siguen vigentes, entre ellas la Comisión Nacional de Evaluación de la Educación Superior (CONAEVA), los Comités Interinstitucionales para la Evaluación de la Educación Superior (CIEES), el Padrón de Excelencia del Posgrado de CONACYT, el Sistema de Acreditación Institucional de la Federación de Instituciones Mexicanas Particulares de Educación Superior (FIMPES), el Centro Nacional de Evaluación de la Educación Superior (CENEVAL) y el Consejo para la Acreditación de la Educación Superior (COPAES).

Por su parte, el Programa Nacional de Educación (PNE) 2001-2006 se basó en tres principios fundamentales: 1. Educación para todos, 2. Educación con calidad, 3. Educación de vanguardia, en donde un elemento considerado para alcanzar logros de buena calidad es contar con las instalaciones e infraestructuras suficientes y en buen estado.

Posteriormente, el Programa Sectorial de Educación 2007-2012, con relación a la infraestructura en la educación superior, menciona que se debe fortalecer, mejorar, ampliar y modernizar la infraestructura y el equipamiento de las instituciones a partir de esquemas de financiamiento y apoyo al trabajo de los cuerpos académicos mediante la concurrencia de fondos federales y estatales, así como impulsar la adecuación de la infraestructura de las instituciones, para atender las necesidades de los alumnos con capacidades diferentes. $^{22}$

Es importante considerar que en la búsqueda por mejorar la eficiencia de los servicios educativos y bibliotecarios, las instituciones se han visto inmersas en una cultura de evaluación que les permite demostrar el buen empleo de los recursos obtenidos, así como justificar la necesidad de seguir invirtiendo en este rubro. Por eso diferentes organismos gubernamentales, principalmente derivados de las políticas educativas (algunos ya mencionados) y de la Asociación Nacional de Universidades e Instituciones de Educación Superior (ANUIES), han instaurado instrumentos de acreditación y certifi-

22 Programa Sectorial de Educación 2007-2012. Disponible en http://promep.sep.gob.mx/infgene /prog_sec.pdf 
cación que incluyen aspectos relacionados con la infraestructura física de las bibliotecas, como son: ${ }^{23}$

- Los Comités Interinstitucionales para la Evaluación de la Educación Superior, A. C. (CIEES), que se integran por nueve cuerpos de pares académicos del más alto nivel de las instituciones de educación superior de todo el país con la misión fundamental de evaluar las funciones y los programas académicos que se imparten en las instituciones educativas que lo solicitan y formular recomendaciones puntuales para su mejoramiento, contenidas en los informes de evaluación, que se entregan a los directivos de las instituciones. ${ }^{24}$

La Tabla Guía de Autoevaluación señala con respecto a la biblioteca:

Rubro 47. Biblioteca

I. Instalaciones

Adecuación de la biblioteca y salas de lectura, y cómo se ajustan a las necesidades del programa educativo, en cuanto a su acondicionamiento y capacidad:

a) de las instalaciones;

b) del mobiliario, la iluminación, la ventilación y la temperatura, y de las adaptaciones para personas con capacidades diferentes.

- Como evidencia el punto 47.1 solicita los planos o croquis de las instalaciones. $^{25}$

- El Consorcio de Universidades Mexicanas (CUMEX), creado a principios del año de 2005, se define como un espacio común para la educación superior de buena calidad en el país y opera en ocho áreas del conocimiento. ${ }^{26}$ En materia de Infraestructura, el Apartado 4 establece 12 indicadores que permiten realizar un diagnóstico de los espacios físicos, por ejemplo, nivel de temperatura en zonas de trabajo, lectura y estantería abierta, nivel de iluminación del área de lectura, nivel de iluminación de área de acervo, intensidad del ruido ambiental, instalaciones para personas con capacidades diferentes, entre otros elementos. ${ }^{27}$

- El Consejo para la Acreditación de la Educación Superior (COPAES), instaurado en el año 2000; en la primera década de su existencia dependía de los CIEES. Sin embargo, en 2010 se separa orgánica y estruc-

23 Compilación realizada por Juan Ángel Vázquez Martínez y presentada en el XIX Encuentro de Bibliotecarios de la península de Yucatán (22 de febrero de 2013).

24 Comités Interinstitucionales para la Evaluación de la Educación Superior, A. C. Disponible en http://www.ciees.edu.mx/

25 CIEES, "Tabla Guía de Autoevaluación", pp. 33 y 83. 
turalmente a fin de articular el quehacer de las diferentes instancias de evaluación y acreditación existentes y concreta la creación de un Sistema Nacional de Evaluación, Acreditación y Certificación de la Educación Superior. $^{28}$

Dentro de los aspectos que evalúa este Consejo en la Tabla Guía de variables e indicadores, el punto 6.3 destinado a la biblioteca señala cuestiones sobre la capacidad de espacio, mobiliario y si se tienen adaptaciones para personas con capacidades diferentes, aspecto que se retoma en el punto 9.1 sobre Infraestructura. En este último apartado, en la Categoría VII, el rubro de Infraestructura remite al Apartado 41. Servicios y acervo de la biblioteca, que en su Indicador 41.1 refiere si la biblioteca cuenta con instalaciones propias, acordes con los servicios que proporciona (solicita como evidencia planos, croquis y fotografías de las instalaciones), mientras que en el Indicador 41.5 refiere a si la capacidad de la biblioteca es adecuada a las necesidades de espacio de los usuarios (la evidencia solicitada son estudios de capacidad instalada y espacios de servicios). ${ }^{29}$

Atendiendo a la premisa de que el cambio es lo único constante en las sociedades actuales, mantenerse informado de las políticas gubernamentales, educativas y otras instancias nacionales e internacionales es una actividad permanente en el caso de la construcción de bibliotecas, que permitirá atender adecuadamente las necesidades emergentes que se presentan y que atañen directamente a las actividades que se realizan en las bibliotecas, como es el caso del Programa Nacional de Auditoría Ambiental (PNAA) que promueve la Procuraduría Federal de Protección al Ambiente (PROFEPA) con gobiernos locales, empresas, asociaciones empresariales, instituciones académicas, auditores ambientales y la entidad mexicana de acreditación (EMA) para mejorar, a través de la auditoría, el desempeño ambiental de los sectores económicos para ir más allá de lo que establecen las leyes en la materia. ${ }^{30}$

En la actualidad, el Instituto Nacional de Estadística y Geografía (INEGI) reporta que hasta el año 2012 la cifra estimada de bibliotecas públicas, especializadas y escolares en el país era de 13 308, pero es difícil conocer las características de construcción de estos edificios, ya que no se encuentran

28 Consejo para la Acreditación de la Educación Superior (COPAES). Disponible en http://www. copaes.org.mx/FINAL/inicio.php

29 COPAES, Tabla Guia de variables e indicadores, p. 51.

30 Procuraduría Federal de Protección al Ambiente (PROFEPA), Programa Nacional de Auditoría Ambiental. Disponible en: http://www.profepa.gob.mx/innovaportal/v/26/1/mx/programa_ nacional_de_auditoria_ambiental.html 
disponibles datos sobre los espacios que ocupan. ${ }^{31}$ Un acercamiento al tema lo ha realizado desde hace tres décadas el CONPAB-IES a través de la elaboración de los diagnósticos sobre los servicios bibliotecarios que brindan las bibliotecas en este sector, en donde se ha compilado información sobre el número, situación, problemática y prospectivas que tienen los edificios que ocupan las respectivas entidades académicas que conforman el Consejo. Aun cuando no se ha logrado consolidar un instrumento definitivo, los esfuerzos realizados representa un gran avance.

El primero de los estudios realizados fue dado a conocer en $1994^{32}$ y coincide con el publicado posteriormente en el año 2000 respecto a la problemática que aquejaba a los edificios, misma que se relacionaba con la aplicación del modelo establecido por el CAPFCE, que no consideraba las variables climáticas y costumbres regionales, entre otros aspectos; así como la escasez de especialistas en la construcción y habilitación de edificios; la insuficiencia de espacios y los problemas relacionados con la humedad, la elevada temperatura, la escasa protección contra el ruido y las pocas posibilidades de ampliación. Es así que de 33 instituciones participantes en el diagnóstico se detectó que 355 bibliotecas contaban con espacios adaptados y 175 habían sido construidos ex profeso. ${ }^{33}$

Posteriormente, en el Diagnóstico CONPAB 2005, 29 instituciones de educación superior que brindaron información reportaron una base de 385 unidades de información de las cuales el $50.5 \%$ eran edificios construidos ex profeso. ${ }^{34}$ En este estudio se percibe un incremento en el mejoramiento físico de las unidades con respecto a lo reportado en el año 2000, los problemas detectados para atender en los próximos años eran los siguientes: capacidad de espacio conforme al ritmo de crecimiento, control de acceso y mecanismos de seguridad, además de que se prevé un decremento en la asistencia física debido al desarrollo tecnológico aplicado principalmente en el uso de los servicios de información. ${ }^{35}$ Además, se identificó que la biblioteca universitaria más antigua es la Biblioteca Pública Universitaria de la UASLP,

31 Instituto Nacional de Estadística y Geografía (INEGI), Patrimonio Cultural y Bibliotecas. Disponible http://www3.inegi.org.mx/Sistemas/temasV2/Default.aspx?s=est\&c=21702

32 J. A. Arellano Rodríguez, J. J. Piña Marquina, H. Valdez, Diagnóstico de los servicios bibliotecarios de las universidades públicas estatales de México, p. 174.

33 J. A. Arellano Rodríguez, J. A. Verdugo Sánchez, Situación de los servicios bibliotecarios de las universidades públicas estatales de México, pp. 70 y 74.

34 En este conteo se realiza la aclaración de que diversas universidades presentaron datos integrados, aun cuando contaran con varias bibliotecas, "[...] como la Universidad de Guadalajara con más de 17 bibliotecas centrales y 26 centros de documentación; y las Universidades de Guanajuato y Autónoma de Nuevo León con alrededor de 40 unidades bibliotecarias cada una”. Diagnóstico de los Sistemas Bibliotecarios de las Instituciones de Educación Superior participantes en el CONPAB-IES, p. 15. 
fundada en 1877, y se constató que el periodo en donde hubo un incremento en el establecimiento de bibliotecas fue el de 1970 a 1990.

Según los avances del diagnóstico nacional bibliotecario de 2012 realizado por el mismo Consejo, en 36 universidades del país se tienen registradas 1279 bibliotecas, de las cuales 409 se han construido ex profeso; destacan en este rubro la Universidad de Guadalajara (UdeG) y la Universidad de Nuevo León (UANL), como se observa en el Cuadro 1.

Cuadro 1. Edificios construidos ex profeso para bibliotecas en las universidades mexicanas

\begin{tabular}{|l|c|c|c|}
\hline \multicolumn{1}{|c|}{ Universidad } & $\begin{array}{c}\text { Total de } \\
\text { bibliotecas }\end{array}$ & $\begin{array}{c}\text { Edificios construidos ex } \\
\text { profeso para bibliotecas }\end{array}$ & $\begin{array}{c}\text { Edificios } \\
\text { adaptados }\end{array}$ \\
\hline Universidad de Guadalajara (UdeG) & 164 & 94 & 70 \\
\hline Universidad de Nuevo León (UANL) & 82 & 63 & 19 \\
\hline Universidad Michoacana (UMICH) & 49 & 6 & 43 \\
\hline $\begin{array}{l}\text { Universidad Autónoma del Estado de México (UAE- } \\
\text { MEX) }\end{array}$ & 60 & 24 & 36 \\
\hline Universidad Autónoma de Tlaxcala (UAT) & 20 & 20 & 0 \\
\hline Universidad Autónoma de San Luis Potosí (UASLP) & 20 & 15 & 6 \\
\hline Universidad de Sonora (USON) & 21 & 15 & 11 \\
\hline $\begin{array}{l}\text { Universidad Juárez Autónoma del Estado de Du- } \\
\text { rango (UJED) }\end{array}$ & 26 & 15 & 6 \\
\hline
\end{tabular}

Fuente: Juan Ángel Vázquez Martínez, Las normas para edificios de bibliotecas y archivos.

Un aspecto a resaltar en este tema es que la diversidad de tipos y conceptos de bibliotecas que existen en el país hacen más compleja la labor de diagnóstico. Aun cuando el INEGI divide las bibliotecas en Escolar (educación básica y media superior), Especializada (instituciones culturales, comerciales, industriales, educativas, bancarias o de investigación, excluidas las universidades) y Pública, en el ámbito universitario se identificaron como tipos de bibliotecas las de investigación, nivel medio superior, posgrado y superior según los diagnósticos realizados por el CONPAB, así como las siguientes denominaciones: bibliotecas centrales, centros de documentación, bibliotecas públicas universitarias y unidades bibliotecarias. De esta forma, cada universidad establece sus denominaciones propias, como ejemplo, en la UASLP los nombres empleados para denominar a las unidades de información que conforman el Sistema de Bibliotecas son Biblioteca Pública Universitaria, Biblioteca Virtual, Centros de Información, Centros Integrales de Aprendizaje y Bibliotecas de Centros Departamentales e Institutos. ${ }^{36}$ 
Las normas bibliotecarias pueden ser definidas como:

Criterios por los que los servicios bibliotecarios pueden ser medidos y valuados. Son determinados por bibliotecarios profesionales a fin de alcanzar y mantener los objetivos que se han propuesto. Las normas pueden ser interpretadas de diferentes maneras como patrón de un ideal, un procedimiento, un modelo, una medida estimativa, un estímulo para el desarrollo y el mejoramiento futuro, y como un instrumento para ayudar en la decisión y en la acción, no sólo a los mismos bibliotecarios, sino a los legos directamente relacionados con la planeación y administración de los servicios bibliotecarios de la institución. ${ }^{37}$

En este sentido, las asociaciones educativas y profesionales han sido un referente importante para el establecimiento de bibliotecas y servicios de información. Es así como desde su creación en 1950 la ANUIES ha jugado un papel importante en la formulación de políticas y directrices en el ámbito de la educación superior y en materia bibliotecaria, ya que esta asociación ha incluido el tema en programas, proyectos y estrategias a seguir, como son la Planeación de la Educación Superior en México (1978), el Plan Nacional de Educación Superior (1981), el Programa Nacional de Educación Superior (1985) y el Programa Integral para la Educación Superior (1986). En este último se destaca la idea de mejorar las condiciones financieras de las instituciones para evitar, entre otras cuestiones, el deterioro de la infraestructura y el rezago en equipos y bibliotecas. ${ }^{38}$ Derivado de este programa se generó el proyecto Fomento a los Servicios Bibliotecarios con la finalidad de definir criterios para la evaluación de las necesidades de incremento cuantitativo y cualitativo del acervo en las IES. ${ }^{39}$

Una acción sumamente importante de la ANUIES para el ámbito bibliotecario fue la adopción en 1968 de las Normas para el Servicio Bibliotecario en las IES de la ABIESI como una forma de impulsar los servicios bibliotecarios y de información. Las normas fueron creadas con el fin de proporcionar a los directores del servicio bibliotecario universitario pautas para orientar sus actividades y facilitar a las autoridades responsables de la educación superior la labor de crear, fomentar y mantener un servicio de tanta trascendencia en el país. Tienen su base en las normas emitidas por la Association of College and Research Libraries (ACRL) de la American Library Association (ALA) publicadas en 1958. Estas normas se reeditaron en 1984. 
En el rubro 2. Estructura y Funcionamiento, punto 2.8, las normas de ABIESI de 1968 aluden a que las colecciones deberán estar concentradas en un solo edificio, a menos que las distancias entre los diferentes locales dificulten la concentración. La centralización se recomienda para reducir costos tanto en la duplicación de obras como de catálogos y en personal especializado; en el rubro 3. Presupuesto, punto 3.4, menciona que los gastos de mantenimiento y adquisición de equipo, así como la construcción de edificios o ampliación de los existentes, deberán formar parte del presupuesto general de la universidad. Asimismo, dedica el rubro 6 al edificio, el equipo y el mobiliario.

Importante es mencionar la participación de asociaciones como ABIESI y AMBAC en seminarios realizados sobre el tema de arquitectura bibliotecaria, como Planeación Arquitectónica de Bibliotecas, organizado por la Asociación Mexicana de Bibliotecarios A. C., la Secretaría de Obras Públicas (SOP), la Secretaría de Educación Pública (SEP) y el Consejo Nacional de Ciencia y Tecnología (CONACYT) en Atlihuetzia, Tlaxcala, del 15 al 17 de junio de 1973, y el Seminario de Arquitectura para Bibliotecas realizado en Morelia, Michoacán, del 6 al 8 de septiembre de 1979 bajo la organización de la Universidad Michoacana y la ABIESI.

En 1978, bajo la premisa de que el desarrollo de un país depende de la generación y aplicación de conocimientos e información, se considera que los servicios de información constituyen un instrumento de progreso individual y social, en tanto que la transferencia de información fomenta la construcción de conocimientos, un ejemplo de lo cual es que en los sectores educativos, económicos, tecnológicos, comerciales e industriales, UNESCO publica el $\mathrm{Ma}$ nual para sistemas y servicios de información, con la finalidad de reunir y ver en perspectiva los conceptos básicos para su planificación y funcionamiento.

El trabajo fue elaborado por Atherton con la documentación disponible en esa época, en donde se establece que para que sean utilizados el conocimiento y la información deben tomarse en cuenta factores como los medios de comunicación y transferencia, así como los espacios físicos destinados para ofertar los servicios informativos que se ofrecen en todo tipo de unidades de información: Salas de Lectura, Bibliotecas, Centros de Información, Centros de Documentación, Archivos, entre otros. De esta forma, la infraestructura de la información que se refiere a la capacidad de hacer que el conocimiento y la información sean accesibles para asegurar su transferencia, y en consecuencia lograr que el conocimiento funcione, consta de siete elementos, entre ellos el de Instalaciones y Equipo.

Precisamente, el capítulo 7. Instalaciones y equipo, punto 7.1 Locales: diseño, ubicación, distribución de espacios, brinda los principios básicos para 
tomar en cuenta estos rubros específicamente en el diseño de nuevos edificios o reestructurar cualquier local que para ser utilizado requiera de un cuidadoso estudio de las funciones que debe cumplir, las cuales parten de dos parámetros básicos: los grupos a servir y las operaciones de información que permiten servir a éstos o que pueden surgir en el futuro como resultado de los progresos tecnológicos previstos y de la evaluación práctica o de los planes a largo plazo. ${ }^{40}$

Más adelante, derivado del diagnóstico realizado por PRODENASBI y con la colaboración de la Dirección de Edificios de la SEP, en 1984 se publican los Indicadores para Bibliotecas Públicas, con la intención de que:

Estos indicadores relativos a diferentes aspectos deseables en las bibliotecas públicas pueden servir de base para desarrollar las tan necesarias bibliotecas públicas no como meros depósitos de libros viejos, sino como verdaderos centros vivos que mejoren, con base a la palabra impresa, la calidad de vida de los habitantes de todos los municipios de la patria. ${ }^{41}$

Es así como a partir de la revisión de las normas internacionales y con las adecuaciones que se consideraron pertinentes y acordes con el contexto nacional, se realizó una propuesta con base en el número de lectores a atender, las plantas arquitectónicas, y los indicadores sobre capacidad, acervos, áreas físicas, dimensiones de terrenos, mobiliario y equipo, recursos humanos, mantenimiento y comodidad, lo que derivó en una propuesta que incluía siete tipos de proyectos de bibliotecas. ${ }^{42}$

También en el año de 1984 se crea el CONPAB-IES como grupo y se genera un escenario de cooperación para analizar la problemática general de los sistemas bibliotecarios afiliados y proponer ante las instancias correspondientes la instrumentación de políticas, lineamientos y normas nacionales que coadyuven al desarrollo de dichos sistemas. Entre sus aportaciones se encuentran las guías y diagnósticos realizados a través de los años, que tienen la finalidad de contextualizar y proponer acciones acordes con la realidad nacional.

Documentos como la Guía metodológica para evaluar las bibliotecas de las instituciones de educación superior de la Región Centro Occidente de ANUIES o los diagnósticos referidos más adelante en el presente escrito permiten dar cuenta de los avances obtenidos en el tema. En 2005 el Consejo emite las Normas para Bibliotecas en Instituciones de Educación Superior e Investigación como apoyo a las IES en su misión de incrementar la calidad de la educación superior y para propiciar que las instituciones optimicen sus recursos y esta-

40 Pauline Atherton, Manual para sistemas y servicios de información.

41 Indicadores para Bibliotecas Públicas, p. 9.

42 Ibid. 
blezcan un lenguaje común, necesario para la colaboración, además de brindar una aproximación cuantitativa y cualitativa para evaluar la efectividad de una biblioteca académica y de promover el uso de medidas de insumos, productos y resultados, en el contexto de la misión de cada institución.

Estas normas, a pesar de no estar reconocidas por ANUIES, vienen de alguna forma a sustituir las emitidas por ABIESI en 1968. Es importante señalar que aun cuando las normas están realizadas conforme a las necesidades de las IES, debido a la carencia de otros lineamientos semejantes son utilizadas también como referente para otro tipo de bibliotecas como las de educación media superior. Con relación al tema de infraestructura, las normas lo mencionan en el rubro 4. Recursos Financieros y de Infraestructura, y además incluyen un anexo denominado Lineamientos para el tratamiento del acervo histórico universitario, en el cual hacen referencia a los espacios físicos destinados a estos acervos, como es el punto 2.1 Alojamiento y espacio.

Los avances y transformaciones científicas y tecnológicas hacen necesaria la actualización, es así como CONPAB publica una segunda edición de las normas en 2012, en la cual se reorganizan y agregan rubros. Por ejemplo, se separa el rubro de Recursos Financieros del de Infraestructura y se agrega el de Organización Técnica de Recursos Documentales. Los cambios de rubros en las diferentes normas emitidas se muestran en el Cuadro 2.

Cuadro 2. Categorías de las normas de ABIESI y CONPAB

\begin{tabular}{|c|l|l|l|}
\hline Rubros & \multicolumn{1}{|c|}{ ABIESI 1968 } & \multicolumn{1}{c|}{ CONPAB 2005 } & \multicolumn{1}{c|}{ CONPAB 2012 } \\
\hline 1 & La función de la biblioteca & $\begin{array}{l}\text { Función de la biblioteca } \\
\text { académica }\end{array}$ & $\begin{array}{l}\text { Función de la biblioteca } \\
\text { académica }\end{array}$ \\
\hline 2 & $\begin{array}{l}\text { La estructura y funcionamien- } \\
\text { to del servicio bibliotecario }\end{array}$ & Organización & Organización \\
\hline 3 & El presupuesto & Recursos Humanos & Recursos Humanos \\
\hline 4 & El personal & $\begin{array}{l}\text { Recursos financieros y de } \\
\text { Infraestructura }\end{array}$ & Infraestructura \\
\hline 5 & $\begin{array}{l}\text { Los recursos documentales } \\
\text { de la biblioteca }\end{array}$ & Colecciones & Recursos financieros \\
\hline 6 & $\begin{array}{l}\text { El edificio, el equipo y el } \\
\text { mobiliario }\end{array}$ & Servicios & Acervos \\
\hline 7 & Los servicios & Educación de usuarios & $\begin{array}{l}\text { Organización técnica de } \\
\text { recursos documentales }\end{array}$ \\
\hline 8 & Evaluación de servicios & Evaluación & Servicios \\
\hline 9 & & & Formación de usuarios \\
\hline 10 & & & Evaluación \\
\hline
\end{tabular}

Fuente: ABIESI, Normas para el servicio bibliotecario; Normas para el Servicio Bibliotecario en Instituciones de Enseñanza Superior e Investigación, ABIESI-SEP; CONPAB-IES, Normas para Bibliotecas en Instituciones de Educación Superior e Investigación (2005 y 2012). 
"Al considerar a las universidades en general como agentes sociales dinámicos, éstas se encuentran expuestas a la influencia de los cambios actuales que se presentan en sus entornos internos, externos y su relación con la sociedad actual, los cuales podrían tener repercusión en su estructura y gestión". ${ }^{43}$ A lo largo de los años, diferentes miembros de las comunidades bibliotecarias y académicas han generado y propuesto normas que satisfacen las necesidades relacionadas con la oferta informativa que ofrecen las bibliotecas y el contexto en que se encuentran inmersas.

Al considerar que se vive un momento propicio para el establecimiento y sostenimiento de un sistema de bibliotecas públicas en la República mexicana, Gordillo Gordillo propone en 1977 la adopción de normas mínimas que guíen todos los aspectos de su implantación y administración, basadas en las Normas para los servicios bibliotecarios: estudio internacional editado por la UNESCO en 1975 y en el discurso de toma de posesión del entonces presidente J. López Portillo. Con referencia al apartado 6. Edificio y Equipo, las recomendaciones se basan en los criterios de eficiencia, flexibilidad interna y posibilidad de expansión que deben de tener los edificios. ${ }^{44}$

Por su parte, Orozco elabora las "Normas para la planeación de edificios para bibliotecas universitarias" con la finalidad de facilitar la consulta rápida para todos los involucrados en el área, pero bajo la observación de que no son normas rígidas y que deben adaptarse según las circunstancias de cada entidad. Bajo el enfoque arquitectónico Orozco incluye los apartados 1. Fórmulas generales; 2. Usuarios; 3. Servicios; 4. Iluminación; 5 . Temperatura, humedad y ventilación; 6. Aspectos estructurales; 7. Espacio Arquitectónico. ${ }^{45}$

En el Programa de Necesidades del Edificio de la Biblioteca de El Colegio de México (1976), primera de una serie de seis obras, Garza Mercado estudia y propone los diferentes aspectos que deben considerarse en la planeación de espacios bibliotecarios. Aportaciones como Función y forma de la biblioteca universitaria (1977), Elementos de planeación administrativa para el diseño arquitectónico (1984) y Guía de lecturas sobre planeación de edificios para bibliotecas (2000) se convierten en referente obligado para los interesados en el tema.

En 1980, la Dirección General de la Universidad Nacional Autónoma de México (UNAM) publica las Normas para bibliotecas universitarias, traduc-

43 Sánchez Vanderkast, op. cit., p. 51.

44 Roberto A. Gordillo Gordillo, "Normas para el establecimiento y sostenimiento de bibliotecas públicas en la República Mexicana”.

45 José Orozco Tenorio, "Normas para la planeación de edificios para bibliotecas universitarias". 
ción autorizada de Standards for University Libraries elaboradas por un comité mixto del College and Research Libraries News y avalado por la American Library Association en 1979. Con relación al edificio de la biblioteca, estas normas brindan orientación sobre los aspectos que debe tener, como ser de dimensión y calidad adecuadas, ser atractivo, acogedor y cuidadosamente diseñado, incluyendo los aspectos ambientales, y priorizar la funcionalidad y ubicación de sus instalaciones. No se indican aspectos cuantitativos.

Hacia mediados de la década de 1990, el Sistema de Bibliotecas de la UASLP, en una etapa de auge en la construcción y remodelación de espacios bibliotecarios, elaboró las Normas para Bibliotecas Universitarias basadas en las elaboradas por Orozco Tenorio y Garza Mercado, las cuales sirvieron de guía para la construcción de las bibliotecas con las que actualmente cuenta la Universidad. En esta fase, gracias a las gestiones realizadas por el entonces rector, Jaime Valle Méndez, la UASLP se convierte en la primera universidad en el país en construir un edificio para biblioteca con un diseño propio, ya que hasta ese momento se construían bajo el modelo del CAPFCE.

Relevantes son los estudios realizados en materia de construcción bibliotecaria, como el Modelo para la evaluación integral de las Bibliotecas de Educación Superior de México (2000) ${ }^{46}$ y los trabajos de titulación presentados en diferentes universidades mexicanas de los cuales se pueden obtener valiosas referencias bibliográficas, entre ellos Recomendaciones para el planeamiento y construcción de bibliotecas universitarias de Javier Arías Velázquez, en 1980; Higiene y Seguridad en bibliotecas públicas de la ciudad de San Luis Potosí, tesis de licenciatura presentada en 1985 por Rosa María Martínez Rider; Aspectos a considerar en el diseño interno de un local o edificio de bibliotecas, tesis de licenciatura de la UNAM en 1990 por parte de Lilia Islas Sánchez; El plan de necesidades como berramienta en la planeación de edificios para bibliotecas universitarias de Daniel Villanueva Rivas en 2004; La aplicación de las normas de seguridad en las bibliotecas de la UNAM construidas en el periodo 19891997 de María Leticia Rivera Ramos también en 2004, y el Plan de prevención de desastres naturales en bibliotecas de Julia Alcibar Hermosillo en 2008.

Otros trabajos destacados en la materia fueron los siguientes:

- Memorias de la elaboración de normas para espacios físicos de las bibliotecas del sistema bibliotecario de la UNAM de María Esther Mendoza Zavala en 1998, referente importante de información sobre el tema dado que el análisis detallado que realiza de las normas internacionales sigue

46 J. A. Arellano Rodríguez, Modelo para la evaluación integral de las Bibliotecas de Educación Superior de México. 
vigente. En esta obra, la autora establece la importancia que tienen las normas como herramientas de auxilio para la planeación de espacios, la construcción de edificios y la implementación de nuevos servicios, y destaca las pocas contribuciones que existen sobre el tema en nuestro país, a diferencia de la vasta literatura que deviene de experiencias internacionales. Son interesantes sus señalamientos y prospectivas realizados sobre el impacto tecnológico en el uso de los espacios y servicios bibliotecarios y muy adecuados para tomarlos en cuenta hoy en día.

- Evaluación de normas para la planeación de edificios de bibliotecas, de 2006, en el cual Ligia del Rosario Ancona analiza la normativa mexicana relacionada con la planeación de edificios poniendo énfasis en los elementos climáticos, especialmente en zonas cálido-húmedas, aspecto escencial dada la diversidad territorial y climática del país.

- Contribución bibliotecológica al diseño de los edificios para bibliotecas académicas, trabajo de tesis para la obtención del grado de maestría (1995), y Diseño de Edificios para Bibliotecas Públicas (2000), son ambas aportaciones de Catalina Naumis Peña. Esta última obra se convierte en un referente obligado por ser de los pocos trabajos que abordan el tema de las bibliotecas públicas del país, además del amplio análisis que realiza en los cinco capítulos en que se divide el libro, los cuales abordan los temas y pasos que deben seguirse desde la elaboración del proyecto hasta la construcción del edificio. Naumis enfatiza el papel que el bibliotecario debe asumir en cada fase para sostener el necesario intercambio de información con los arquitectos. El capítulo tres, Arquitectura y normas para los edificios de bibliotecas, describe los conceptos principales que deben tomar en cuenta las distintas áreas de la biblioteca con base en la normativa internacional, así como las condiciones de iluminación, ventilación, limpieza, señalética y otros aspectos.

Posteriormente, Naumis participó en la Reunión sobre Infraestructura Bibliotecaria para la Preservación y Rehabilitación de Espacios de la Red Nacional de Bibliotecas Públicas, que se llevó a cabo dentro del 8o. Congreso Nacional de Bibliotecas en Tuxtla Gutiérrez, en el 2008, evento en que se volvió a tomar el tema en el ámbito de las bibliotecas públicas.

\section{Problemática detectada}

La década de 1970 representa la pauta para la edificación de bibliotecas en el 
de las normas que se refieren a su planeación, diseño y construcción. Si bien en un principio y de manera lógica éstas se basaron en la normativa internacional, en este periodo se realizaron importantes aportaciones que incluían un acercamiento al contexto nacional, como son los trabajos de ABIESI, AMBAC, Orozco Tenorio y Garza Mercado.

Sin embargo, en las décadas subsecuentes no hubo aportaciones significativas, con el resultado de que la normativa utilizada para el diseño de bibliotecas se encuentra desfasada con respecto a las necesidades actuales, ya que se siguen teniendo como base las emitidas por ABIESI en 1968, por lo que se requiere de una revisión profunda que permita la inclusión de aspectos que van desde la prevención de factores de riesgo en las instalaciones hasta el cumplimiento de la normatividad federal vigente en materia de construcción de obras públicas.

Por su parte, la diversidad de bibliotecas o unidades de información que forman parte de los sistemas bibliotecarios públicos y universitarios ocasiona que sea complejo aplicar una normativa única para todas ellas, por lo que en la revisión y actualización de la normativa para la construcción, remodelación y adaptación de espacios bibliotecarios se debe considerar la capacidad de adaptación a cada contexto o, en su caso, reflexionar sobre si se deben emitir normas como tales o sólo confeccionar guías, manuales y lineamientos con la suficiente flexibilidad que permita incluir las normativas relacionadas y los aspectos emergentes que se presenten.

Se requiere un marco legal que fortalezca a las bibliotecas y clarifique los diferentes tipos y apoyos que éstas requieren, de tal forma que no se encuentren a merced de los cambios gubernamentales. Al respecto, en la década de 1970, Gordillo Gordillo resaltó el importante e imprescindible papel que juegan las asociaciones en la planeación y mejoramiento de los servicios bibliotecarios a partir del establecimiento de normas, las cuales en diversos países han precedido siempre la promulgación de leyes en el área. Es innegable que para obtener resultados exitosos en el establecimiento de una normativa eficiente, las asociaciones profesionales son las más indicadas para conformar equipos interdisciplinarios que además de aunar la experiencia de los bibliotecólogos, involucren a arquitectos, ingenieros y a las entidades legislativas correspondientes para brindar un amplio panorama de acción acorde al contexto nacional y a las tendencias internacionales. ${ }^{47}$

Importante es señalar que aun con la experiencia lograda en el país en las últimas cuatro décadas en materia de construcción de bibliotecas, tanto en el ámbito público como en el privado (en donde se cuentan con importantes edificaciones construidas para tal fin) no se cuenta con datos que se refieran 
al número y las características de estos espacios, por lo que se hace imprescindible la realización de un censo para obtener esta información.

Resultado de lo anterior es la poca literatura que existe sobre las experiencias obtenidas en la construcción, ampliación, remodelación y adaptación de estos espacios que permitan la consulta y toma de decisiones sobre el tema. Como bien establece Naumis Peña, muchas de las bibliotecas en el país no cuentan con la documentación respectiva sobre el desarrollo de los procesos de construcción-remodelación de sus archivos, así como tampoco con los planos desarrollados ni en original ni en copia. ${ }^{48}$

Por último, es también preocupante que las normas actuales presenten una deficiente citación de los documentos originales en los que se basaron y de los cuáles obtuvieron los indicadores establecidos.

\section{Conclusiones}

En una sociedad en permanente movimiento, la biblioteca se convierte en un agente activo para acceder a la información a través de los servicios que oferta, además de una alternativa para la convivencia y la expresión social, por lo que la planeación y el diseño de espacios seguros, funcionales y estéticos requiere de una normativa actualizada y pertinente que brinde las bases para poder ofrecer una gama de servicios de información tanto presenciales como virtuales que promuevan el autoaprendizaje, el interés por la lectura, la curiosidad de investigar y la interacción social de las comunidades.

Aspectos impostergables son también la consideración de aquellos elementos que permitan la integración de comunidades vulnerables, como las personas con alguna discapacidad física, así como los aspectos relacionados con el uso sustentable de los recursos bibliotecarios debido a los efectos que el cambio climático tiene en todos los países del mundo, los cambios tecnológicos y la seguridad nacional, entre otros aspectos no contemplados en la normativa actual.

Es un momento propicio para retomar el tema dada la experiencia práctica que se ha desarrollado en las últimas cuatro décadas, pero si no se toman las medidas correspondientes en la materia, se corre el riesgo de que instancias ajenas a la profesión sigan estableciendo parámetros e indicadores con perspectivas impropias para el quehacer bibliotecario, como ya acontece en los procesos de gestión de calidad, certificación y acreditación a los cuales están sujetos los sistemas bibliotecarios nacionales. 
Por último, el establecimiento de una normativa sobre arquitectura bibliotecaria debe constituirse dentro de un amplio esquema de consultas, diagnósticos e investigaciones cuantitativas y cualitativas que permitan una crítica constructiva y la propuesta de alternativas viables de aplicación para los diferentes esquemas bibliotecarios que existen en nuestro país, y que a su vez permitan la inclusión de temas emergentes, aspecto común de las sociedades actuales.

\section{BibliogRAfía y FUENTES CONSULTADAS}

Ancona Martínez, L. R., Evaluación de normas para la planeación de edificios de bibliotecas. Propuesta de indicadores y criterios para bibliotecas universitarias, tesis de maestría, México, UNAM, Bibliotecología y Estudios de la Información, 2006.

Ancona Riestra, Roberto, "Biblioteca Estatal de Ciencias. Parque Científico y Tecnológico de Yucatán”, en Impacto académico y social de la construcción de espacios en los servicios de información de Bibliotecas y Archivos, San Luis Potosí, México, 2014, pp. 57-79.

Arellano Rodríguez, J. A., Modelo para la evaluación integral de las Bibliotecas de Educación Superior de México, México: SEP, Subsecretaría de Educación Superior/ENBA, Dirección General de Educación Superior, 2000. (Serie Temas bibliotecológicos)

__; Piña Marquina, J. J.; Valdez, H., Diagnóstico de los servicios bibliotecarios de las universidades públicas estatales de México, Mérida: UADY/CONPAB, 1994. (Mecanografiado)

—_ Verdugo Sánchez, J. A., Situación de los servicios bibliotecarios de las universidades públicas estatales de México, México: SEP/ENBA, 2000. (Serie Temas bibliotecológicos)

ABIESI (Asociación de Bibliotecarios de Enseñanza Superior y de Investigación), Normas para el Servicio Bibliotecario, Xalapa, Veracruz, 1968.

Atherton, Pauline, Manual para sistemas y servicios de información, París: UNESCO, 1978.

Biblioteca Central. Libros, muros y murales. 50 aniversario, Celia Martín Marín (ed.), México: UNAM/DGB, 2006.

CIEES (Comités Interinstitucionales para Evaluar la Educación Superior), "Tabla Guía de Autoevaluación”, en Metodología general para la evaluación de programas de educación superior a distancia, México, 2009, 111 pp. 
CONPAB-IES (Consejo Nacional para Asuntos Bibliotecarios de las Instituciones de Educación Superior, A. C.), Guía para evaluar bibliotecas de instituciones de educación superior e investigación, $\mathrm{La}$ Paz, Baja California Sur, México, 2012, 43 pp. (Colección Normatividad)

—_ Normas para Bibliotecas de Instituciones de Educación Superior e Investigación, 2a. ed., La Paz, Baja California Sur, México, 2012, 52 pp. (Colección Normatividad)

—_ Normas para Bibliotecas en Instituciones de Educación Superior e Investigación, Guadalajara, Jalisco: Comisión Permanente de Normatividad 2004-2006, 2005, 46 pp.

COPAES (Consejo para la Acreditación de la Educación Superior), Tabla Guía de variables e indicadores, México, 2010.

CUMEX (Consorcio de Universidades Mexicanas), Indicadores de Bibliotecas y Centros de Información del CUMEX, México, 2010, 18 pp.

Diagnóstico de los Sistemas Bibliotecarios de las Instituciones de Educación Superior participantes en el CONPAB-IES, México: CONPAB/ SEP, 2005.

Duarte Jáquez, Ricardo, La Universidad que queremos... Una propuesta de trabajo para consolidar la calidad académica y la mejora de la gestión en el periodo rectoral 2012-2018. Resumen, Ciudad Juárez, Chihuahua: UACJ, 2012, 27 pp.

Fernández de Zamora, R. M., "Construcción de Nuevas Bibliotecas en México, 1981”, en Memorias de las XIII Jornadas de Biblioteconomía (3 al 7 de mayo, Hermosillo, Sonora), 1982, pp. 327-333.

__ " "Las bibliotecas y sus edificios en México: Una mirada histórica”, en Celia Mireles Cárdenas (coord.), Arquitectura bibliotecaria en México, San Luis Potosí: UASLP, 2012, pp. 57-75.

Garza Mercado, A., Función y forma de la biblioteca universitaria. Elementos de planeación administrativa para el diseño arquitectónico, 2a. ed., México: COLMEX, 2003, 194 pp. (Jornadas, 83)

_- Programa de necesidades para la ampliación de la biblioteca de El Colegio de México 2004-2024, México: COLMEX, 2006, 63 pp.

Gordillo Gordillo, Roberto A., "Normas para el establecimiento y sostenimiento de bibliotecas públicas en la República Mexicana", en Memorias de las VIII Jornadas Mexicanas de Biblioteconomia (1 al 6 de mayo de 1977, Guadalajara, Jalisco), México: AMBAC, 1977, pp. 117-152.

Indicadores para Bibliotecas Públicas, pres. de Ana María Magaloni, México: Dirección General de Publicaciones y Bibliotecas/SEP, 1984.

Latimer N.; Niegaard, H., IFLA Library Building Guidelines: Developments Reflections, München: K. G. Saur, 2007. (IFLA Series on Bibliographic Control)

Leyes federales vigentes. Disponibles en: http://www.diputados.gob. $\mathrm{mx} /$ LeyesBiblio/ 
Martínez Rider, Rosa María, Higiene y Seguridad en bibliotecas públicas de la ciudad de San Luis Potosí, tesis de licenciatura, México, UASLP, Escuela de Biblioteconomía, 1985.

Mendoza Zavala, María Esther, Memoria de la elaboración de normas para espacios físicos de las Bibliotecas del Sistema Bibliotecario de la UNAM, tesis de licenciatura, México, ENBA, 1998.

Mireles Cárdenas, C. (coord.), Arquitectura bibliotecaria en México, San Luis Potosí, México: UASLP/ECI, 2012, 231 pp.

_- Impacto académico y social de la construcción de espacios en los servicios de información de bibliotecas y archivos, San Luis Potosí, México: UASLP/ECI, 2014, 172 pp.

Murguía Jáquez, Laura P. et al., "Influencia de la infraestructura bibliotecaria en el desarrollo de producción científica en universidades mexicanas inmersas en procesos de acreditación”, en Revista General de Información y Documentación, vol. 23, núm. 2, 2013, pp. 333-367. Disponible en: http://revistas.ucm.es/index.php/RGID/ article/view/333-367

Naumis Peña, Catalina, "Definición de espacios arquitectónicos para bibliotecas académicas", en Revista General de Información y Documentación, vol. 10, núm. 2, 2000, pp. 135-165.

—- Contribución bibliotecológica al diseño de edificios para bibliotecas académicas, tesis de maestría, México, UNAM, 1995, 335 p.

—_, Diseño de edificios para bibliotecas públicas, México: Consejo Nacional para la Cultura y las Artes/Dirección General de Bibliotecas, 2008.

Normas para el Servicio Bibliotecario en Instituciones de Enseñanza Superior e Investigación, ABIESI-SEP, 1984, 11 pp.

Normas y directrices para bibliotecas universitarias y cientificas, Madrid: REBIUN CRUE, Ministerio de Educación y Cultura, Dirección General del Libro, Archivos y Bibliotecas, 1999. (Última modificación: 12 de octubre de 2010)

Orozco Tenorio, José, "Normas para la planeación de edificios para bibliotecas universitarias", en Seminario de Arquitectura para Bibliotecas (6 al 8 de septiembre de 1979, Morelia, Michoacán), México: ABIESI, 1980.

PRODENASBI. Programa de Desarrollo Nacional de los Servicios Bibliotecarios y de Información, vol. I, México: Bibliotecas públicas, SEP-CONESCAL, 1980. (Descripción general del estudio)

Sánchez Vanderkast, Egbert J. (coord.), La naturaleza objetiva y subjetiva de las Políticas de Información, México: IIBI/UNAM, 2012, 238 pp.

— , Políticas de Información en las Universidades Públicas Estatales, México: IIBI/UNAM, 2010, 354 pp.

Seminario de Arquitectura para las Bibliotecas (6 al 8 de septiembre de 1979, Morelia Michoacán), México: ABIESI, 1980.

Standards for Libraries in Higher Education, Association of College \& Research Libraries, 2011. Disponible en: http://www.ala.org/acrl /standards/standardslibraries\#principles 
Universidad Autónoma de San Luis Potosí, Sistema de Bibliotecas, Normas para Bibliotecas Universitarias, s. a. Documento inédito.

Universidad Nacional Autónoma de México, Dirección General de Bibliotecas (DGB), Normas para Bibliotecas Universitarias, México, 1980, 35 pp.

Vázquez Martínez, Juan Ángel, Las normas para edificios de bibliotecas y archivos. Disponible en: http://www.slideshare.net/CBUADY/ las-normas-para-edificios-de-bibliotecas-y-archivos

Villar Rubio, Manuel Fermín, "Impacto social de los servicios bibliotecarios en la Universidad Autónoma de San Luis Potosí”, en Impacto académico y social de la construcción de espacios en los servicios de información de Bibliotecas y Archivos, San Luis Potosí, México, 2014, pp. 13-31.

, "Infraestructura de Unidades de Información de la UASLP en la Ciudad capital y el interior del Estado", en Segundo Seminario sobre Servicios de Información. Arquitectura de bibliotecas y archivos en México. Impacto Académico y Social, 2012. 